9-19-2016

\title{
Using School Improvement and Implementation Science to Integrate Multi-Tiered Systems of Support in Secondary Schools
}

\author{
Hank Bohanon \\ Loyola University Chicago, hbohano@luc.edu \\ C. Gilman \\ B. Parker \\ C. Arnell \\ G. Sortino
}

Follow this and additional works at: https://ecommons.luc.edu/education_facpubs

Part of the Education Commons

\section{Author Manuscript}

This is a pre-publication author manuscript of the final, published article.

\section{Recommended Citation}

Bohanon, Hank; Gilman, C.; Parker, B.; Arnell, C.; and Sortino, G.. Using School Improvement and Implementation Science to Integrate Multi-Tiered Systems of Support in Secondary Schools. Australasian Journal of Special Education, 40, 2: 99-116, 2016. Retrieved from Loyola eCommons, Education: School of Education Faculty Publications and Other Works, http://dx.doi.org/10.1017/jse.2016.8

This Article is brought to you for free and open access by the Faculty Publications and Other Works by Department at Loyola eCommons. It has been accepted for inclusion in Education: School of Education Faculty Publications and Other Works by an authorized administrator of Loyola eCommons. For more information, please contact ecommons@luc.edu.

\section{c) (†) $\ominus$}

This work is licensed under a Creative Commons Attribution-Noncommercial-No Derivative Works 3.0 License.

(C) The Authors 2016 
Bohanon, H., Gilman, C., Parker, B., Amell, C. and Sortino, G. (2016) 'Using School

Improvement and Implementation Science to Integrate Multi-Tiered Systems of Support in

Secondary Schools', Australasian Journal of Special Education, , pp. 1-18. doi: 10.1017/jse.2016.8.

Australasian Journal of Special Education, Year; Volume(Issue): 1-36

Manuscript ID: AJSE-SEP-16-0019.R1

Running header: School Improvement, Implementation, and MTSS

Approximate word count: 9,201 words

Keywords: MTSS, response to intervention, secondary schools, positive behavior support, school improvement, implementation science

ORIGINAL ARTICLE

Using School Improvement and Implementation Science to Integrate

Multi-Tiered Systems of Support in Secondary Schools*

Hank Bohanon', Carrie Gilman², Ben Parker², Chris Amell², and Gabe

Sortino $^{2}$

${ }^{1}$ Loyola University of Chicago, Chicago, IL, USA

${ }^{2}$ Northfield Middle School and High School, Northfield, VT, USA

*This manuscript was accepted under the Editorship of Michael Arthur-Kelly.

Correspondence: Hank Bohanon, School of Education, Loyola University of Chicago, 820

N. Michigan Ave., Chicago, IL 60611, USA. Email: hbohano@luc.edu 
School Improvement, Implementation, and MTSS

\begin{abstract}
The purpose of this paper is to describe the integration of tiered interventions and supports in secondary schools, sometimes referred to as multi-tiered systems of support (MTSS). The interventions include academic, behavioural, social, and emotional supports for all students. A description of the connections across specific MTSS systems, datasets, and practices is discussed. The article addresses the implementation of MTSS through the lens of school improvement and implementation science. A case example of a school implementing MTSS is provided to highlight the strengths and challenges of MTSS in secondary settings.
\end{abstract}


The purpose of this paper is to describe the integration of tiered intervention supports in secondary school settings. Schools place an emphasis on academic, behavioural, emotional, and social supports, the goal being to prevent failure for students at risk. The interventions include data-driven, evidence-based solutions for struggling students. However, these approaches are not always aligned. Schools are making efforts to provide a cohesive framework for intervention in schools through multi-tiered systems of support (MTSS). In this paper we provide a discussion of the assimilation of support systems through school improvement and implementation science. It also provides a case example of this integration at the secondary school level.

\section{Academic and Behaviour Problems in Secondary Schools}

Problem behaviour can decrease access to adequate instruction for secondary students. For example, each disciplinary action for a student requires up to 20 minutes of the instructor's time. The period for addressing a discipline referral goes up to 45 minutes for administrators (Scott \& Barrett, 2004). Over time, responding to discipline results in lost educational experience for students. It also distracts school leaders from their focus on instruction. For example, one Midwest state in America reported losing 1,000,000 instructional days in one year due to discipline problems (Voices of Youth in Chicago Education, 2014).

Discipline actions also disproportionally impact groups of students based on ethnicity. Disproportionate outcomes around discipline become apparent as early as preschool. For instance, African American students represent 18\% of the overall enrollment in the United States, but represent $48 \%$ of the proportion of students with one or more out-of-school suspensions. Suspensions from school occur for $5 \%$ of Caucasian students and $16 \%$ of all African American students (U.S. Department of Education Office for Civil Rights, 2014). However, negative outcomes for students are not limited to behaviour and ethnicity. 
Students with disabilities, who are 16 or older, are twice as likely drop out of high school as their peers without a diagnosed disability. Once these students leave high school, they have a lower eventual high school completion rate than their peers without disability (80\% compared to 90\%; Chapman, Laird, Ifill, \& KewalRamani, 2011). Yet, with proper educational supports, students with disabilities can have improved postsecondary outcomes. These outcomes include employment, access to postsecondary education and job training, and the ability to develop friendships, live independently, and manage a savings account (Newman et al., 2011). Successful completion of secondary school is a catalyst for positive outcomes, but graduation requires interventions that support student success. One approach to improve these outcomes is that of MTSS.

\section{Individual MTSS Approaches}

MTSS systems are based on three tiers of support (Gamm et al., 2012). These tiers include primary interventions that are provided to the entire school population, secondary supports that focus on individuals or groups of students with common needs, and tertiary supports that provide individualised treatments (Walker et al., 1996). Interventions can range from community- to school-based approaches (Walker, 2010). MTSS share common characteristics related to effective implementation and sustainability of evidenced-based practices. Some of these factors include obtaining support for the model (e.g., from administration, staff, financial), strong teams that guide implementation and represent diversity of the setting, effective training and coaching, program alignment (e.g., with goals, philosophy), high visibility of outcomes to all stakeholders (e.g., using data for decisions), refraining from using labels for students within the system (e.g., tertiary kids), culturally relevant programs, and addressing change in behaviour of staff and administration (Forman, Olin, Hoagwood, Crowe, \& Saka, 2009; Lueck \& Kelly., 2010). Furthermore, MTSS approaches typically include shared, measurable, and explicitly stated goals; efficient and 
effective processes for identifying or referring students for connection with evidenced-based practices (EBPs); and system-level commitments (e.g., school and district level

administrative support). One MTSS for behaviour that includes these components is schoolwide positive behaviour interventions and supports (SWPBIS).

Schoolwide positive behaviour interventions and supports. SWPBIS is 'a framework or approach comprised of intervention practices and organizational systems for establishing the social culture, learning and teaching environment, and individual behaviour supports needed to achieve academic and social success for all students' (Sugai et al., 2010, p. 13). SWPBIS includes supports across three tiers. The first level involves universal supports such as identifying and explicitly teaching expectations, acknowledging appropriate behaviour, redirecting inappropriate behaviour (e.g., promoting appropriate behaviours, re-teaching desired behaviours), and monitoring overall student performance (Horner, Sugai, \& Anderson, 2010; McIntosh, Goodman, \& Bohanon, 2010). Secondary-level supports include focused instruction and feedback for groups of students, and increased academic and behavioural structure (Horner et al., 2010; McIntosh, Bohanon, \& Goodman, 2010a). Finally, tertiary-level supports include interventions based on the function or purpose of the problem behaviour and the individualised needs of the student (Gettinger \& Stoiber, 2006; Horner et al., 2010; McIntosh, Bohanon, \& Goodman, 2010a).

SWPBIS teams develop core behavioural norms and curriculum (Reinke, Herman, \& Stormont, 2013) and support instructional staff in delivering effective instruction that is relevant, rigorous, and well paced (Simonsen, Fairbanks, Briesch, Myers, \& Sugai, 2008). Instruction that decreases the aversive nature of academic work would be included at this level (e.g., providing choice of task, task variation, decreasing task difficulty; Munk \& Repp, 1994). As will be discussed, this work is based on the assumption that behaviour and 
instruction are related (Munk \& Repp, 1994; Weeks \& Gaylord-Ross, 1981). Another type of MTSS related to academic support is response to intervention (RtI).

Response to intervention. RtI is 'the practice of providing high quality instruction and interventions matched to student need, monitoring progress frequently to make decisions about changes in instruction or goals and applying student response data to important educational decisions’ (Kurns \& Tilly, 2008, p. 1). Within RtI, teams screen for students who are failing or at risk of failure, monitor progress for students at risk of failure, use empirically validated practices and instruction across tiers, and organise instructional resources to have the greatest impact on all students (Burns \& Gibbons, 2012). As with SWPBIS, there is a focus on effective core instruction. For example, components of effective teaching of reading instruction (e.g., teaching phonemic awareness, phonics instruction) should guide the selection and implementation of core academic curriculum. Teachers' instruction should also be guided by the features of effective practices, including meaningful engagement with language and explicit teaching of skills (Kurns \& Tilly, 2008). The role for leadership teams addressing RtI includes analysing school behaviour and achievement data; identifying required changes to the current staff training, tools, and supports; and monitoring fidelity of implementation of instruction (Burns \& Gibbons, 2012). Students can also be taught skills to increase the likelihood of their success in the core curriculum (e.g., self-regulated learning strategies; Sawyer, Graham, \& Harris, 1992). Another MTSS approach that especially focuses on increasing self-regulation skills for all students is that of social and emotional learning (SEL).

Social and emotional learning. SEL is 'a process for helping children and even adults develop the fundamental skills for life effectiveness. SEL teaches the skills we all need to handle ourselves, our relationships, and our work, effectively and ethically' (Collaborative for Academic, Social, and Emotional Learning, 2007, p. 1). As a tiered approach, SEL is 
concerned with addressing improvements in social and emotional skills, attitudes towards others and self, positive behaviours, reducing problems related to conduct and emotional distress, and enhancing academic performance (Durlak, Weissberg, Dymnicki, Taylor, \& Schellinger, 2011).

These outcomes are addressed by systematically teaching behavioural, affective, and cognitive skills to increase abilities related to social awareness, self-awareness, responsible decision-making, relationship skills, and self-management (Collaborative for Academic, Social, and Emotional Learning, 2003). Cognitive skills include thinking or conceptual maps that help students approach their work and relationships. These can include learning to break down tasks, reflecting on one’s own behaviour performance, and learning to provide selfacknowledgment for reaching a goal. Similar to SWPBIS, students within this model can be taught through direct instruction to engage in respectful dialogue with others. In SEL, there is additional emphasis on teaching all students to be self-determined through personal decisionmaking and self-evaluation. Goals for SEL leadership teams include increasing student social competence in ways that will simultaneously improve their academic and social development (Durlak et al., 2011). Specifically, SEL teams infuse skills into the core curriculum, including self-monitoring (Norris, 2003), goal setting, literature that addresses conflict management, and effective instructional strategies (Zins \& Elias, 2007). For example, students can be taught to self-evaluate their progress on a writing assignment using a checklist or rubric. Although different researchers developed each MTSS approach, they each share common elements of systems, practices, and data.

\section{Commonalities Across MTSS}

Each MTSS previously mentioned is concerned with developing, adopting, and implementing a core curriculum that is evidenced based. Each approach requires similar systems (e.g., resources, teams, administrative support), practices (e.g., requirements for selecting EBPs), 
and data (e.g., screening, progress monitoring, evaluation). Additionally, all three approaches recommend obtaining stakeholder feedback about the process prior to, during, and after intervention (Bohanon \& Wu, 2014; Elias, Zins, Graczyk, \& Weissberg, 2003; Kurns \& Tilly, 2008; Hall, n.d.).

Schools that have implemented specific schoolwide MTSS approaches have demonstrated significantly higher desirable outcomes (e.g., social and emotional skills, behaviour, academic performance) than schools that did not (Durlak et al., 2011). Schools that effectively implement schoolwide approaches to behaviour also have demonstrated improvements in academic outcomes (e.g., standardised reading; Bradshaw, Mitchell, \& Leaf, 2010; Bradshaw \& Pas, 2011; Childs, Kincaid, \& Peshak George, 2010) and behavioural performance (Bradshaw et al., 2010). There would appear to be common themes in terms of systems, practices, and data across each MTSS system. However, without some type of catalyst for integration, carrying them out can be daunting. Conceptualising MTSS approaches through the lens of school improvement may enhance integration of these separate but related approaches.

\section{Conceptualising MTSS Approaches Together}

MTSS implementation (Gamm et al., 2012) may be enhanced if it is implemented with a school improvement-by-design approach (Rowan, Correnti, Miller, \& Camburn, 2009; Rowan \& Miller, 2007). A hallmark of improvement by design is collaboration between local educational agencies and outside organisations (e.g., special education state professional development grants) to implement new approaches. Within this arrangement, the most effective design principles tended to be those focused on the ways schools organise in preparation for the instructional changes envisioned by the program or approach.

School improvement-by-design principles involve controlling contextual factors of the school during implementation (Rowan et al., 2009). The first principle involves 
encouraging cultural control of the vision and mission by connecting them with the innovation. For example, if a school is interested in a social and emotional intervention, their school mission and vision should include some reference to nonacademic student domains. The second principle includes addressing procedural control of the organisational systems. For example, if an innovation requires professional development, the school's calendar should reflect the time needed to provide staff instruction. The third stage involves organising the professional control of the practices and staff workflow. For instance, if school staff are required to teach behavioural expectations, this act should not detract from their ability to provide academic instruction.

The key components of school improvement by design (i.e., cultural, procedural, professional controls) can be used to help integrate MTSS frameworks across approaches (e.g., SWPBIS, SEL). Proper alignment of staff culture, procedures, and professional roles has been associated with increases in cognitive, intrapersonal, and interpersonal growth for students (Pellegrino \& Hilton, 2013). Rather than implementation being driven based on professional roles, mandates, or funding sources, innovations are guided by goals specific to the school. Subsequently, tasks for team members are aligned with goals for school improvement. Professional roles are then aligned to tasks (Alin, Maunula, Taylor, \& Smeds, 2013) that lead to the implementation of the school improvement plan. Helping schools consider MTSS through a school improvement approach may be enhanced through the science of innovation and implementation.

\section{Implementing Innovations Through Stages}

MTSS school teams put evidenced-based practices into action. The application of these practices can occur in stages that can require several years of effort (Blasé, Fixsen, Sims, \& Ward, 2015). These stages may address reasons why innovations tend to fail (Kotter, 1995). Implementation science focuses on understanding how innovations are adopted for short- and 
long-term success. Schools that have considered components of implementation science in their implementation of MTSS have shown greater fidelity and improved behavioural outcomes compared to schools that did not (Bohanon \& Wu, 2014). The application of MTSS through implementation science and innovation may enhance schools’ ability to approach it as a school improvement effort.

Within a combined implementation science (Blasé et al., 2015) and innovation model (Kotter, 1995), innovations follow a series of stages. Stage 1, exploration and adoption, involves creating a sense of urgency for the need to change on the part of the staff. During this stage, buy-in for an initiative is enhanced through highlighting the necessity for the intervention (e.g., examining existing discipline data for patterns). It also includes connecting proposed innovation with the schools' vision and mission.

Stage 2, program installation, typically involves creating a powerful coalition, or team, to guide the approach. These teams should be prepared to function as an effective group and be provided with clear role designations (e.g., preparing data for meetings, communication with staff). The third stage, initial implementation, could include piloting components of the intervention with small groups (e.g., grade levels, departments). The purpose of these pilots is to provide local examples of the approach that can be shared with the staff. The fourth stage, full operation, might include changing the professional roles for the entire staff. For example, every staff member could become responsible for teaching some component of the local behaviour expectations to their students.

During the fifth stage, innovation, the team guiding the intervention uses their increasing credibility with the staff to change additional systems and practices. Schools could add additional intensive interventions based on the success of their Tier 1 approaches. Also, staff roles would become codified during this stage to include their role in the approach within their position descriptions. Within the sixth stage, sustainability, training is provided 
to potential new leadership team members. Training new staff to assume leadership roles allows for approaches to survive changes in school personnel. This stage also can involve communication to the entire school community (e.g., students, staff, parents) that connects the school's success to the innovation. Although steps appear linear, they may in fact be iterative (Blasé et al., 2015; Kotter, 1995). For example, a school might be in Stage 6 in their schoolwide efforts, but in Stage 1 for their Tier 2 work.

In summary, MTSS, when applied individually, can improve outcomes for students at risk of failure. However, staff may need to harness the power of school improvement by design and implementation science to integrate these supports effectively. The following section provides a case study that illustrates the integration of MTSS approaches.

\section{Case Example}

The case example comes from Northfield Middle and High School (NMHS), located in rural Vermont. The community’s population includes significant generational poverty, as well as military and academic families connected to a local military college. The school includes 31 certified teachers and 295 students. Ninety-six percent of the students are Caucasian and have generally low mobility (11\%) and dropout rates (3\%). Fourteen percent of the students in Grades 6-8, and 24\% of students in Grades 9-12 qualify as having some type of a disability. Thirty-nine percent of the students are considered to be impoverished based on qualifying for free or reduced-price meals.

Systems, Data, and Practice

The MTSS implementation approach of NMHS includes a variety of systems, data, and practices.

Systems. The systems at NMHS related to MTSS include (a) teams to guide implementation, (b) administrative support, (c) review of current initiatives, and (d) the development plans for action. NMHS has an overall school improvement leadership team that 
guides the implementation of MTSS. This team includes administrators, general education teachers, and special educators who meet weekly. Their mission is to provide Tier 1 supports, and ensure capacity to address Tier 2 and 3 interventions to students as needed. At each meeting, the team uses data sheets (described in the data section of this paper) to screen for the effectiveness of the core curriculum and identify students who are in need of more intensive support. The initial analysis includes universal screening data for all students. Although the MTSS leadership team meets weekly, formal review of universal screening data takes place three times per year.

The first data review meeting involves examining academic, behavioural, and social and emotional information (e.g., universal screening data). Formative benchmark assessment data are compared to students’ grades to identify strengths and weaknesses. Interventions that are in place are reviewed for fidelity in order to determine if students are responding. If the intervention was not implemented with fidelity, it is impossible to determine if the student is benefiting from the approach. When a lack of fidelity of implementation is determined, support is provided to the teachers through coaching. Adaptations to the intervention are also made, as needed, to improve contextual fit. The second meeting takes place following the next wave of assessments during the following quarter. Data are reviewed to determine if students are making expected progress towards benchmarks established during quarter one. The final meeting takes place after the third quarter assessments and includes students' final grades.

NMHS uses a teacher advisor action planning process (TAAP) as a bridge between Tiers 1 and 2 services. The mission of the TAAP team is to determine if students are truly in need of additional supports beyond Tier 1 , based on progress monitoring and implementation fidelity data. The membership of the TAAP team changes for each individual student and can include a teacher advisor, parents or guardians, the identified student, a Tier 2 team 
representative, the referring teacher, related services (e.g., special education, nurse), and other members as required. A key member of the team is the teacher advisor. Each teacher is assigned between 10 to 14 students from across grade levels to be a part of their advisory group. The purpose of these advisory groups is to ensure that all students have at least one adult in the building who meets with them on a regular basis. Teacher advisors can serve as student advocates as needed.

A TAAP meeting is conducted when (a) the universal data indicate a need, or (b) a teacher has a concern about a student who has not already been identified for Tier 2 or Tier 3 interventions, or (c) a teacher believes a student needs more support beyond tier one. A teacher advisor then calls a TAAP meeting and gathers relevant student data to help the team determine if more intensive supports are required.

The educational support team (EST) currently directs supports for students in Tiers 2 and 3. The teams consist of the student's teacher advisor and a standing group of team members. These team members have been trained in problem-solving approaches to intervention that are based on the function of the student's behaviour (e.g., attention, escape, avoidance). The function of the student's behaviour is initially determined through interviews and review of behavioural screening data, attendance, and academic performance data. The mission of this team is to provide intensive supports to students based on entrance criteria, monitor student progress, and review fidelity of plan implementation. The most intensive tier three supports are provided by groups of specialised professionals (e.g., school counsellors, special education teachers) but are available to all students regardless of diagnosis or disability. These supports are developed based on additional diagnostic data and intensive intervention approaches.

Practices. Practices for MTSS include prevention and remediation at each tier. These can include approaches that address academic performance, behavioural functioning, and 
social emotional learning. For example, upon determining that a significant number of students were not responding to their universal academic curriculum, NMHS identified a Tier 1 intervention for all students. This intervention consists of an online core curriculum instructional program (Study Island) that is connected with their formative benchmarking data. The software provides flexible instruction based on each student's performance. All students in the middle school are scheduled for a 49-minute block with the system every other day. For students who still require additional support, Tier 1 plans are developed.

Tier 1 plans generally run for 9 weeks and are reviewed for student progress and implementation fidelity (e.g., task analysis of steps for the intervention) every 3 weeks. The plans are developed by grade-level data teams and overseen by the teacher advisors. Universal Tier 1 interventions are selected for the students based on a common intervention protocol. The protocol includes information describing how each of the interventions aligns with the possible function of students’ problem behaviour (e.g., escape, avoidance) and referring criteria (e.g., office discipline referrals [ODRs], issues with classwork). Tier 1 interventions can include improved home communication, homework club, differentiated instruction, preferential seating, and organisational help such as a daily planner.

The team then completes an online Tier 1 form that consists of the student's goals, interventions, and progress monitoring information. Next, ideas that have been generated are shared with parents through email. Teachers who submit Tier 1 support requests are prompted every 3 weeks to review student progress and check for implementation fidelity. Although those interventions expire after 9 weeks, new ones can be developed based on progress monitoring data. Students are referred to Tier 2 interventions if they do not make sufficient progress based on their Tier 1 supports, or the team decides more intensive strategies are required. 
The EST develops Tiers 2 and 3 intervention approaches. These plans also run for a timespan of 9 weeks and are reviewed every 3 weeks to monitor student progress and to check for implementation fidelity. These are based on specific protocols that allow staff to map potential approaches to student needs. Similar to Tier 1 strategies, students are also assigned to interventions based on the function of their behaviour and referring criteria (e.g., ODRs, team referral). Interventions include approaches such as check-in and check-out, homework club, skill development, and a hands-on experiential program that allows students to achieve credit for courses in community environments (e.g., gardening, community service).

Data. The data used in MTSS implementation include fidelity data for tracking, treatment integrity, and program development. Implementation fidelity for MTSS is guided by Vermont's MTSS field guide and self-assessment, which is based on current MTSS research (Vermont Reads Institute at UVM and Vermont Statewide Steering Committee on RTII, 2014). The guide identifies components of MTSS implementation including (a) developing a systematic and comprehensive approach to intervention, (b) effective collaboration, (c) providing high-quality and differentiated instruction, (d) using comprehensive and balanced assessment systems, and (e) developing local expertise. A statesupported MTSS external coach provides feedback to the staff concerning the selfassessment.

SWPBIS fidelity of implementation is guided by the Benchmarks of Quality ([BOQ], Kincaid, Childs, \& George, 2010). The BOQ has been found to be a reliable (overall a = 0.96; test-retest between Pearson product-moment $=0.94, p<.01$; interrater reliability Pearson product-moment $=0.87, p<.01$ ), valid, and efficient tool for measuring the universal level of positive behaviour supports (Childs et al., 2010). It is completed annually in the spring of each year by the MTSS leadership team and an external MTSS coach. 
Student information is used at each tier to identify those who are not responding to interventions. Data decision rules are created to help team members decide how students move in and out of tiers. NMHS uses a core dataset to make initial decisions for problem identification across each tier. These include formative assessments and summative data. These datasets involve assessments of academic performance, grades, discipline, and measures of SEL. Common Core Measures of Academic Progress (MAP) data from the Northwest Evaluation Association (NWEA) are used as the universal assessment tool to inform instruction in both English language arts (ELA) and mathematics for all students.

MAP testing is administered to all students three times per academic year. The data are used in conjunction with the Student Risk Screening Scale (SRSS) to help identify students who are at risk academically, behaviourally, socially, and emotionally (Lane et al., 2013; Lane, Oakes, Carter, \& Messenger, 2015). Additionally, the SRSS is a universal screening tool also used three times per year to identify students at risk of internalising and externalising behaviour problems. Along with MAP and SRSS data, NMHS also continuously collects student grades in ELA and maths and ODRs. These data are entered into an integrated, online data-planning worksheet (see Figure 1) using Excel.

Data for TAAP, Tier 2, and Tier 3 team meetings include attendance, current grades, assessment scores, brief teacher comments from email, and other relevant information (e.g., ODRs, visits to the school nurse). The TAAP team determines if concerns about students are isolated to one class and could be handled through Tier 1 interventions, or if there are larger issues that needs to be addressed by the Tier 2 team with a full plan.

\section{<<PLEASE INSERT FIGURE 1 ABOUT HERE $>>$}

Data decision rules. The data sheets reviewed by the universal team are conditionally formatted by colour to identify students who are high performing, on-grade-level performance, partially proficient, and low performing. Red denotes students who are low 
performing in academics and have high-risk ratings in behaviour. Any student with two or more red categories receives a more intensive support plan. Students with one red category are discussed at Tier 1 grade-level team meetings, and that team makes a determination regarding whether a plan is needed. The teacher advisors check in at least weekly with relevant adults and students possessing any categories in red.

The colour yellow in any cell denotes partially proficient academic performance and medium risk ratings in behaviour. Teachers and teacher advisors of students in this group are promoted to monitor student progress. The support at this level comes from the classroom teacher, and student progress is reviewed at grade-level team meetings. If the student does not demonstrate progress between data meetings, the teacher advisor, along with the classroom teachers, consider a Tier 1 support plan. The colour green denotes students who are performing at grade level academically and have low-risk ratings for behaviour. The colour blue indicates a student is performing two grade levels above their peers academically. Teachers are made aware that these students need to be challenged in order to advance their educational performance.

Tracking interventions. The data sheets are also colour-coded in the name column to denote if a plan is currently in place for a student. The colour blue implies a student is receiving special education services and has an individualised education plan (IEP). Teachers consult with the student's special educator with concerns for this group. The colour maroon is used for students who are supported by a Section 504 plan from the Vocational Rehabilitation Act of 1973. This plan requires reasonable accommodations for those who do not have an IEP. Orange denotes students who are involved in Tier 2 and 3 plans. These aforementioned systems, practices, and data allow the MTSS team to provide supports to all students. These approaches have been embedded within NMHS school improvement process through stages of implementation. 
Stages of Implementation and School Improvement by Design

The following section addresses how NMHS aligned MTSS with their school improvement process. It includes details outlining how the staff are able to acquire professional control of their mission and vision, educational practices, and professional workflow over the course of six stages of implementation science.

Stage 1: Exploration and adoption stage. The exploration and adoption stage (Blasé et al., 2015) involves creating a vision for MTSS. The vision for NHMS was created following a series of critical events that had prompted a sense of urgency for the school (Kotter, 1995). The federally mandated No Child Left Behind Act ([NCLB], 2002) set yearly progress goals for all schools in the United States. When schools do not meet adequate yearly progress (AYP) based on standardised assessment data, they are placed into a school improvement planning process. At the time, NMHS was among the lowest ranked in the state. The school had also lacked consistent administrative leadership for several years. In 2011, the school hired a principal whose approach was aligned with the vision and mission of the school. During the first year of his leadership, he selected a small group of staff (Kotter, 1995) in the middle school to begin research on the possibility of implementing SWPBIS the following school year.

The school had seen a significant increase in ODRs in the 2012-2013 school year. Specifically, there was a group of 7-10 students that were causing a majority of the discipline problems for the school. The staff became aware that their reactive approaches to discipline were not effective. Based on the sense of urgency generated from a review of the discipline data, the school made the decision to consider committing to SWPBIS.

To establish buy-in, all staff were surveyed and voted unanimously to pursue SWPBIS as a behaviour program. This input allowed the leadership team to create an approach that would work for the school, and also encouraged each member of the group to 
take ownership. The team was sent to a statewide SWPBIS meeting, which allowed them to create a sense of common purpose and culture. Support from administration included hours of paid time for SWPBIS and MTSS meetings, paid stipends and contractual time for SWPBIS and MTSS coordinators, and a budget line item for program implementation.

There were several positive results of the exploration and adoption stage. The team was able to learn from other schools how to focus on prevention of behaviour problems rather than reaction to these occurrences. Crucially, the staff also developed an understanding of the function of behaviour. This understanding allowed the team to see student behaviour as a function (e.g., escape, avoid) rather than a problem inherent to the student. The team also decided to adopt an effective ODR data collection system (i.e., Schoolwide Information System), which increased their capacity to assess student needs. The team also incorporated the school's input while developing their schoolwide expectation framework.

Connection to school improvement. Connections to school improvement at this stage include the staff's ability to shape the mission and vision of the school to align with MTSS. (Rowan et al., 2009; Rowan \& Miller, 2007). The MTSS leadership changed the vision of the school to align with their new approach to school improvement. The new vision stated that NMHS was to become, 'A supportive community whose purpose is to maximize student learning. Students are challenged to realize the value of learning and to reach their potential in a safe, healthy and respectful environment. At NMHS, we are committed to students becoming productive, independent, caring members of our democratic society’.

Stage 2: Program installation stage. A major part of the program installation stage (Blasé et al., 2015) was further developing the MTSS skills of the leadership team (Kotter, 1995). The team uses careful consideration when bringing both new and veteran teachers into the MTSS process. The academic, social, and emotional components of MTSS also require the team to develop new skills. The administration supports and encourages team members to 
attend training on facets of MTSS related to master scheduling and course programing. As a result of this training, the school altered the master schedule to allow for more opportunities for intervention classes and team-teaching through block scheduling. Importantly, these changes were based on the recommendations of the staff, which led to increased ownership in the MTSS process. The administration also changed the role of their planning room coordinator. This role previously focused on more reactive discipline. His new role involved coordinating MTSS, data coaching, and supporting more intensive interventions such as check-in and check-out.

Connection to school improvement. In terms of connections to school improvement at this stage, the MTSS leadership team identified reliable data that allowed them to control the selection of their practices (Rowan et al., 2009; Rowan \& Miller, 2007), identify areas for improvement, and monitor student progress. This included the selection of formative assessments for academics, behaviour, and SEL, as aligned with the tenets of their vision. They developed a streamlined system for organising and interpreting the data through integrated online spreadsheets. This process allowed for the integration of multiple datasets (e.g., formative assessment, grades, ODRs), and the ability to identify students who were in need of additional support. They also selected data that assess the SEL of students (i.e., SRSS).

Stage 3: Initial implementation stage. The initial implementation stage (Blasé et al., 2015) involved creating initial successes that encouraged staff and student participation. The school piloted SWPBIS to create evidence that the approach might work in their setting. The entire faculty and staff took part in a basic training on SWPBIS on the first day of the new school year, and middle school teachers received more intensive training as the year progressed. The use of student recognition was also seen as a major step in the overall process of obtaining student and staff buy-in. This included frequent acknowledgments and 
schoolwide celebrations. Students and staff also established new roles (Kotter, 1995) as part of their involvement in SWPBIS implementation; teachers developed a leadership club to support student participation in SWPBIS, and the students were involved in event planning and schoolwide celebrations. The staff also connected to the community during this stage. The local newspaper began to publish articles about the SWPBIS students of the week, and stores simultaneously began donating gift certificates to be used as prizes.

Connection to school improvement. The initial focus on SWPBIS implementation led to a major decrease in discipline problems and increased instructional time. Specifically, there were reductions in ODRs and suspensions. Behavioural data indicated that by the third year of implementation, $90 \%$ of the students had between zero to one ODR. There were also noticeable reductions in ODRs for major discipline events such as fighting and disorderly conduct. The ODRs per day, per month, per average daily enrolment per 100 students decreased from .36 during 2012-2013, to .29 in 2013-2014, and finally to .20 in 2014-2015. The ODR rates for all students dropped 44\% between years one and three of their SWPBIS implementation. These data reinforced the efforts of the staff towards their goals for school improvement.

Stage 4: Full operation stage. The school is still working towards the full operation stage (Blasé et al., 2015) of MTSS implementation. The success of SWPBIS encouraged the staff to move forward with the broader efforts of MTSS related to academic and social and emotional support. For instance, specific staff moved into new roles of leadership (Kotter, 1995; e.g., MTSS coordinator), and all teachers now use data for decision-making and the development of student supports. The changes in staff roles have allowed for more time to determine if students are responding to interventions at every tier. Students are also able to monitor their own academic progress and set goals for their own learning using the Study Island software. 
Connection to school improvement. As previously mentioned, the MTSS leadership team revised the workflow of their staff (Rowan et al., 2009; Rowan \& Miller, 2007). Prior to MTSS implementation, core instructional teachers dealt with behavioural problems independent of each other. Few data were used to evaluate the needs of students. Under the new MTSS workflow, all teachers became data collectors and intervention decision-makers for their students.

Stage 5: Innovation stage. The initial credibility (Kotter, 1995) of the SWPBIS efforts is allowing the school leadership team to move forward with the innovation stage (Blasé et al., 2015) of MTSS implementation. For example, teacher team meetings and parent teacher conferences now include more objective data. This, in turn, establishes credibility for teachers when meeting with students’ parents. The school also uses this new credibility to begin to select additional datasets outside of typical academic performance measures (e.g., SRSS) to look for internalised behavioural needs for students. The leadership team is able to establish trust from their stakeholders that allows them to collect multiple universal assessments to address school improvement. It is also leads to the selection of additional interventions (e.g., Study Island). The staff simultaneously relies less on traditional tests and quizzes in classes and more on benchmarks of learning (e.g., MAP) to determine student growth.

Connection to school improvement. The control of practices for school improvement (Rowan et al., 2009; Rowan \& Miller, 2007) includes the structural changes in NMHS’s master schedule. These changes ensure that core academic instruction is implemented during the first part of the day. They also incorporate time during the day for all students to have access to Tier 1 intervention.

Stage 6: Sustainability stage. The school is also moving forward with the sustainability stage (Blasé et al., 2015) of MTSS. Positive behavioural improvements have 
occurred at the same time as measurable forms of academic growth. Table 1 presents the changes in academic performance for reading in maths between years. The data are presented based on the students' cohort graduate timeline. The students have demonstrated, on average, a 31\% increase in reading and 11\% increase in maths performance based on MAP testing.

\section{$<<$ PLEASE INSERT TABLE 1 ABOUT HERE $>>$}

Although the NMHS has experienced success, there are several areas of improvement for the school's MTSS. These include solidifying the role of the MTSS coordinator, increasing consistency of professional development related to MTSS to include all staff, and maintaining support of the administration. Due to small staff size, sustainability is a concern. Because of complexity, specific SWPBIS efforts appear to be easier to sustain than the broader MTSS approach. For example, the role of the MTSS coordinator is a staff position, although the school is making efforts to transform this to an administrative role. Staff are attempting to refine the efficacy of interventions at each tier. Additionally, the leadership team is working towards bringing in additional related staff members (e.g., special education teachers, social workers) to the larger MTSS effort. Finally, the school is making an effort to connect MTSS with their elementary feeder school to support student transitions to the middle school.

Connection to school improvement. In terms of controlling practices for school improvement (Rowan et al., 2009; Rowan \& Miller, 2007), the SEL data (i.e., SRSS) allow the staff to determine alternatives to suspension for students at risk. These interventions include seeking guidance from specialists and counsellors to develop support plans. In this way, the staff are able to consider every facet of students' lives (e.g., academic, emotional, social) and provide creative problem-solving to meet needs and execute their school improvement goals.

\section{Discussion}


NMHS is modelling many of the overall components of MTSS. They also carry out specific MTSS-related approaches (e.g., SWPBIS). Both school improvement and implementation science appear to be related to the successful outcomes of the school. Although still a work in progress, NMHS's example can provide useful insights for successful application of MTSS.

In terms of MTSS, the school applies a wide range of interventions (Walker, 2010) that include behavioural (e.g., check-in and check-out), academic (e.g., Study Island), and social (e.g., connecting students to teacher advisors) components. They also are able to obtain support for operation from their local stakeholders. These supporters include administration, staff, students, and community members. Furthermore, they have developed a strong leadership team that is connected to visible outcomes such as student academic and behavioural improvement for the school. They also changed the roles of their staff through aligning their programs and training with their school's mission and vision (Forman et al., 2009; Lueck \& Kelly, 2010).

With regard to SWPBIS, the school implements universal supports for teaching and acknowledging the key behavioural expectations for students (Horner et al., 2010; McIntosh, Goodman, \& Bohanon, 2010). They do so through focused instruction, ongoing feedback for students using data, and increased academic and behavioural structure (Horner et al., 2010; McIntosh et al., 2010a). Student interventions are selected based on the function of the problem for the student through a review of relevant data (Gettinger \& Stoiber, 2006; Horner et al., 2010; McIntosh et al., 2010b).

NMHS'S MTSS alignment of interventions to student needs also mirrors components of RtI. The use of data protocols at advanced tiers appeared to facilitate the match between student needs and supports. The school monitors student progress and implementation fidelity to determine if students are responding to interventions (Burns \& Gibbons, 2012; Kurns \& Tilly, 2008). The school also employs universal screening tools (e.g., MAP, SRSS) 
that are matched to valid practices to address student needs. Additionally, the staff organise systems and interventions at each tier (Burns \& Gibbons, 2012) in order to facilitate consistent implementation by staff.

NMHS also addresses SEL. Specially, the schoolwide expectations address skills broader than respect and responsibility (e.g., personal accountability). The staff also focus on diminishing the likelihood of conduct problems and on enhancing student learning. The use of the SRSS data allows the teams to identify students who are in some type of emotional distress (Durlak et al., 2011). As with SEL implementation, the leadership team connects with their community (e.g., teachers, students) prior to implementing their approaches (Bohanon \& Wu, 2014 Elias et al., 2003; Hall, n.d.; Kurns \& Tilly, 2008).

In terms of the effects of schoolwide implementation of interventions, the NMHS's data reflect improvements in academic functioning (e.g., MAP results; Bradshaw et al., 2010; Bradshaw \& Pas, 2011; Childs et al., 2010) and on behavioural outcomes (i.e., ODRs; Bradshaw et al., 2010). The schoolwide implementation of MTSS may be enhanced by implementation of effective components of school improvement by design. Particularly, the leadership team addresses the cultural control of the vision and mission, the procedural control of the school's organisational systems (e.g., make up of teams), and professional control of the workflow and practices of the staff (e.g., all staff using data for decisions; Rowan et al., 2009). The resulting alignment of the culture, procedures, and professional roles may be a major factor in the improvement of cognitive, intrapersonal, and interpersonal growth for students (Pellegrino \& Hilton, 2013). Furthermore, new staff are hired, in part, based on their support of MTSS. Once employed, they are empowered to make significant decisions about the school's culture.

The catalyst for the successful school improvement may include components of implementation science. Although the stages are not all linear in sequence, they do provide a 
framework for understanding how the school moved from consideration to innovation regarding MTSS (Blasé et al., 2015). A recurring theme throughout each stage is the creation of a sense of urgency for change, and the development of staff for new roles (Kotter, 1995). In tandem, these concepts allow for a continuous improvement and ensure that the staff support interventions.

\section{Limitations}

Although encouraging, there are several limitations for this example. First, the case involves a small school that is situated in a rural area. All of the implications for MTSS from this example may not be applicable to larger school districts. However, the small staff size requires that MTSS be implemented with efficiency based on the commitment of limited resources. NMHS is also able to redefine existing staff roles to support their MTSS and school improvement efforts. Larger systems with greater resources could learn from this example by committing budget line items towards implementation efforts and rethinking current expenditures.

The stages of implementation described in this study would also be replicable to larger systems. NMHS's use of teams to review data would be replicable if bigger schools could focus on smaller divisions within their setting. A school-within-a-school model would allow for teams of teachers to review data for students, make changes to instruction, implement interventions, track students’ progress, and monitor implementation fidelity. Any school could emulate NMHS's use of data to build a sense of urgency for buy-in and also prepare their staff for new roles. The school used existing internal resources to implement MTSS. These findings also could be applicable to international sites.

For instance, this example could possibly apply to other countries and settings with similar levels of community poverty and lack of resources. Likewise, implementation of SWPBIS in Australia and Asia could perhaps be enhanced by considering the intensive 
behaviour supports within a schoolwide context of combined academic, behaviour, and social and emotional interventions. Considering the stages of implementation would also allow schools to create their own local capacity and examples to drive SWPBIS and MTSS in their settings.

\section{Conclusion}

The purpose of this paper was to discuss and provide examples of MTSS implementation in secondary school settings. The school emphasised integrating academic, behavioural, emotional, and social supports. Each of these specific MTSS approaches in the case example is implemented in concert to prevent failure for students at risk. A focus on school improvement may be one way to help teams integrate separate MTSS initiatives (e.g., SWPBIS, RtI) into one cohesive plan. By focusing on the vision, systems, and practices of the school, leadership teams may better align interventions for all students. Consideration of the science of implementation and innovation may help school leadership teams develop buyin, and sustain and innovate their MTSS approaches in ways that benefit all students and staff. Future research should consider ways to monitor the integration and implementation efforts for schools across domains of MTSS in secondary settings. 
School Improvement, Implementation, and MTSS

\section{Acknowledgments}

The authors acknowledge the work of the staff, students, and community members of Northfield Middle School and High School, and Vermont's Multi-Tiered System of Supports Response to Intervention and Instruction Vermont team for the content of this article.

\section{Author Note}

No external funding was used in the development of this manuscript. The co-authors of this manuscript are staff members of the school described in this case. The study is being submitted with the permission from the school's administration. 


\section{References}

Alin, P., Maunula, A. O., Taylor, J. E., \& Smeds, R. (2013). Aligning misaligned systemic innovations: Probing inter-firm effects development in project networks. Project Management Journal, 44(1), 77-93. doi:10.1002/pmj.21316

Blase, K. A., Fixsen, D. L., Sims, B. J., \& Ward, C. S. (2015, April). Implementation science: Changing hearts, minds, behavior, and systems to improve educational outcomes. Paper presented at the Wing Institute’s Ninth Annual Summit on Evidence-Based Education, Berkeley, CA. Retrieved from http://nirn.fpg.unc.edu/resources/implementation-science-changing-hearts-mindsbehavior-and-systems-to-improve

Bradshaw, C. P., Mitchell, M. M., \& Leaf, P. J. (2010). Examining the effects of schoolwide positive behavioral interventions and supports on student outcomes: Results from a randomized controlled effectiveness trial in elementary schools. Journal of Positive Behavior Interventions, 12, 133-148. doi:10.1177/1098300709334798

Bradshaw, C. P., \& Pas, E. T. (2011). A statewide scale up of positive behavioral interventions and supports: A description of the development of systems of support and analysis of adoption and implementation. School Psychology Review, 40, 530548. Retrieved from http://bit.ly/29OQKJS

Bohanon, H. \& Wu, M.-J. (2014). Developing buy-in for positive behavior support in secondary settings. Preventing School Failure, 58, 223-229. doi:10.1080/1045988X.2013.798774

Burns, M. K., \& Gibbons, K. (2012. Implementing response-to-intervention in elementary and secondary schools: Procedures to assure scientific-based practices $\left(2^{\text {nd }}\right)$. New York, NY: Guilford Press. 
Chapman, C., Laird, J., Ifill, N., \& KewalRamani, A. (2011). Trends in high school dropout and completion rates in the United States: 1972-2009 (NCES 2012-006). Retrieved July 11, 2014, from http://nces.ed.gov/pubsearch

Kincaid, D., Childs, K., \& George, H. (March, 2010). School-wide Benchmarks of Quality (Revised). Unpublished instrument. USF, Tampa, Florida

Childs, K. E., Kincaid, D., \& Peshak George, H. (2010). A model for statewide evaluation of a universal positive behavior support initiative. Journal of Positive Behavior Interventions, 12, 198-210. doi:10.1177/1098300709340699

Collaborative for Academic, Social, and Emotional Learning. (2003). Safe and sound: An Educational leader's guide to evidence-based social and emotional learning (SEL) programs. Chicago, IL: Author.

Collaborative for Academic, Social, and Emotional Learning. (2007). CASEL briefs: Background on social and emotional learning. Chicago, IL: Author.

Durlak, J. A., Weissberg, R. P., Dymnicki, A. B., Taylor, R. D., \& Schellinger, K. B. (2011). The impact of enhancing students' social and emotional learning: A meta-analysis of school-based universal interventions. Child Development, 82, 405-432. doi:10.1111/j.1467-8624.2010.01564.x

Elias, M. J., Zins, J. E., Graczyk, P. A., \& Weissberg, R. P. (2003). Implementation, sustainability, and scaling up of social-emotional and academic innovations in public schools. School Psychology Review, 32, 303-319. Retrieved from http://bit.ly/2a3nRuD

Forman, S. G., Olin, S. S., Hoagwood, K. E., Crowe, M., \& Saka, N. (2009). Evidence-based intervention in schools: Developers’ views of implementation barriers and facilitators. School Mental Health, 1, 26-36. doi:10.1007/s12310-008-9002-5 
Gamm, S., Elliott, J., Halbert, J. W., Price-Baugh, R., Hall, R., Walston, D., ... Casserly, M. (2012). Common Core State Standards and diverse urban students: Using multi-tiered systems of support. Retrieved from http://www.eric.ed.gov/ERICWebPortal/search/detailmini.jsp?_nfpb=true\&_\&ERICE xtSearch_SearchValue_0=ED537476\&ERICExtSearch_SearchType_0=no\&accno=E D537476

Gettinger, M., \& Stoiber, K. C. (2006). Functional assessment, collaboration, and evidencebased treatment: Analysis of a team approach for addressing challenging behaviors in young children. Journal of School Psychology, 44, 231-252.

doi:10.1016/j.jsp.2006.03.001

Hall, S. L. (n.d.). Create your implementation blueprint stage 1: Exploration. Retrieved from http://www.rtinetwork.org/getstarted/develop/create-your-implementation-blueprintstage-1-exploration

Horner, R. H., Sugai, G., \& Anderson, C. M. (2010). Examining the evidence base for school-wide positive behavior support. Focus on Exceptional Children, 42, 1-14. Retrieved from http://bit.ly/29Juv6z

Kotter, J. P. (1995). Leading change: Why transformation efforts fail. Harvard Business Review, 73(2), 59-67. Retrieved from http://bit.ly/29X738c

Kurns, S., \& Tilly, W. D. (2008). Response to intervention blueprints: School building level edition. Retrieved from http://www.nasdse.org/Portals/0/SCHOOL.pdf

Lane, K. L., Oakes, W. P., Carter, E. W., \& Messenger, M. (2015). Examining behavioral risk and academic performance for students transitioning from elementary to middle school. Journal of Positive Behavior Interventions, 17, 39-49.

doi:10.1177/1098300714524825 
Lane, K. L., Oakes, W. P., Ennis, R. P., Cox, M. L., Schatschneider, C., \& Lambert, W. (2013). Additional evidence for the reliability and validity of the student risk screening scale at the high school level: A replication and extension. Journal of Emotional and Behavioral Disorders, 21, 97-115. doi:10.1177/1063426611407339

Lueck, C., \& Kelly, M. S. (2010). School based mental health in Illinois: Assessing the present and looking toward the future. Chicago, IL: Illinois Children's Mental Health Partnership.

McIntosh, K., Bohanon, H., \& Goodman, S. (2010a, November). Toward true integration of academic and behavior response to intervention systems. Part two: Tier 2 support. Communiqué, 39(3), 4-6. Retrieved from http://ecommons.luc.edu/education_facpubs/24

McIntosh, K., Bohanon, H., \& Goodman, S. (2010b, December). Toward true integration of academic and behavior response to intervention systems. Part three: Tier 3 support. Communiqué, 39(4), 30-31. Retrieved from http://ecommons.luc.edu/education_facpubs/23

McIntosh, K., Goodman, S., \& Bohanon, H. (2010, October). Toward true integration of academic and behavior response to intervention systems. Part one: Tier 1 support. Communiqué, 39(2), 1, 14-16. Retrieved from http://ecommons.luc.edu/education_facpubs/20/

Munk, D. D., \& Repp, A. C. (1994). The relationship between instructional variables and problem behavior: A review. Exceptional Children, 60, 390-401.

Newman, L., Wagner, M., Knokey, A.-M., Marder, C., Nagle, K., Shaver, D., \& Wei, X. (with Cameto, R., Contreras, E., Ferguson, K., Greene, S., \& Schwarting, M.) (2011). The post-high school outcomes of young adults with disabilities up to 8 years after 
high school: A report from the National Longitudinal Transition Study-2 (NLTS2)

(NCSER 2011-3005). Menlo Park, CA: SRI International.

No Child Left Behind (NCLB) Act of 2001, Pub. L. No. 107

-110 , § 115, Stat. 1425 (2002).

Norris, J. A. (2003). Looking at classroom management through a social and emotional learning lens. Theory into Practice, 42, 313-318. doi:10.1207/s15430421tip4204_8

Pellegrino, J. W., \& Hilton, M. L. (Eds.). (2013). Education for life and work: Developing transferable knowledge and skills in the 21st century. Washington, DC: National Academies Press.

Reinke, W. M., Herman, K. C., \& Stormont, M. (2013). Classroom-level positive behavior supports in schools implementing SW-PBIS: Identifying areas for enhancement. Journal of Positive Behavior Interventions, 15, 39-50.

doi:10.1177/1098300712459079

Rowan, B., Correnti, R., Miller, R. J., \& Camburn, E. M. (2009). School improvement by design: Lessons from a study of comprehensive school reform programs. Philadelphia, PA: Consortium for Policy Research in Education. Retrieved from http://repository.upenn.edu/cpre_researchreports/54/

Rowan, B., \& Miller, R. J. (2007). Organizational strategies for promoting instructional change: Implementation dynamics in schools working with comprehensive school reform providers. American Educational Research Journal, 44, 252-297. doi:10.3102/0002831207302498

Sawyer, R. J., Graham, S., \& Harris, K. R. (1992). Direct teaching, strategy instruction, and strategy instruction with explicit self-regulation: Effects on the composition skills and self-efficacy of students with learning disabilities. Journal of Educational Psychology, 84(3), 340-352. doi:10.1037/0022-0663.84.3.340 
Scott, T. M., \& Barrett, S. B. (2004). Using staff and student time engaged in disciplinary procedures to evaluate the impact of school-wide PBS. Journal of Positive Behavior Interventions, 6, 21-27. doi:10.1177/10983007040060010401

Simonsen, B., Fairbanks, S., Briesch, A., Myers, D., \& Sugai, G. (2008). Evidence-based practices in classroom management: Considerations for research to practice. Education \& Treatment of Children, 31, 351-380. doi:10.1353/etc.0.0007

Sugai, G., Horner, R. H., Algozzine, R., Barrett, S., Lewis, T., Anderson, C., ... Simonsen, B. (2010). School-wide positive behavior support: Implementers' blueprint and selfassessment. Eugene, OR: University of Oregon.

U.S. Department of Education Office for Civil Rights. (2014). Civil rights data collection: Data snapshot (school discipline). Retrieved from https://www2.ed.gov/about/offices/list/ocr/docs/crdc-discipline-snapshot.pdf

Vermont Reads Institute at UVM and Vermont Statewide Steering Committee on RTII (2014). Vermont multi-tiered system of supports response to intervention and instruction (MTSS-RtI) self-assessment. Retrieved from http://www.vriuvm.org/MTSS-RtII/wp-content/uploads/2014/06/MTSS-RtIISelfAssessment-062014.pdf

\section{Vocational Rehabilitation Act of 1973. Public Law 93-112}

Voices of Youth in Chicago Education. (2014). VOYCE's groundbreaking bill, SB 100, to address “school-to-prison pipeline” passes Illinois Legislature [Press release]. Retrieved from http://voyceproject.org/campaigns/campaign-common-sensediscipline/sb100/

Walker, H. M. (2010). Commentary: Facilitating collaborative partnerships with schools: It's where you get it. School Mental Health, 2, 102-103. doi:10.1007/s12310-010-9035-4 
Walker, H. M., Horner, R. H., Sugai, G., Bullis, M., Sprague, J. R., Bricker, D., \& Kaufman, M. J. (1996). Integrated approaches to preventing antisocial behavior patterns among school-age children and youth. Journal of Emotional and Behavioral Disorders, 4, 194-209. doi:10.1177/106342669600400401

Weeks, M., \& Gaylord-Ross, R. (1981). Task difficulty and aberrant behavior in severely handicapped students. Journal of Applied Behavior Analysis, 14, 449-463. doi:10.1901/jaba.1981.14-449

Zins, J. E., \& Elias, M. J. (2007). Social and emotional learning: Promoting the development of all students. Journal of Educational and Psychological Consultation, 17, 233-255. doi:10.1080/10474410701413152 
TABLE 1.

Academic Growth Between Years Based on MAP Assessment Data MAP Cohort Data:

\begin{tabular}{lcccccc}
\hline Graduation & Year & Grade & \% & \% Change & $\%$ & \% Change \\
year & & level & Proficient & between & Proficient & between \\
& & & in maths & years & in reading & years \\
\hline 2019 & Fall 2014 & 8th grade & 40 & & 60 & +10 \\
& Fall 2015 & 9th grade & 78 & +38 & 70 & +8 \\
\hline 2020 & Fall 2014 & 8th grade & 51 & & 67 & \\
\hline & Fall 2015 & 9th grade & 74 & +23 & 75 & +14 \\
\hline & Fall 2014 & 8th grade & 31 & & 53 & \\
\hline
\end{tabular}




\section{FIGURE CAPTION.}

\section{FIGURE 1.}

Data Worksheet for Team Decision-Making. The figure provides an example of the data sheets used as the base level data for team at all three tiers. Students' names are colour coded based on the type of plan they are receiving.

\begin{tabular}{|c|c|c|c|c|c|c|c|}
\hline & \multicolumn{7}{|c|}{ Green-Continue Universal Instruction } \\
\hline Blue-Current IEP & \multicolumn{7}{|c|}{ Yellow-Potential Area of Concern } \\
\hline Orange-Current EST & \multicolumn{7}{|c|}{ 1-Red-Area of Concern } \\
\hline Maroon-Current 504 & \multicolumn{7}{|c|}{$2+$ Red-Need to Put Plan in Place } \\
\hline Green-Tier 1 Plan & De & notes Scores & more Than - & wo Grade Le & vels Above & Proficient Sc & ore \\
\hline \multirow[t]{8}{*}{ Student Name } & ODR Count & $\begin{array}{l}\text { Winter MAP } \\
M \text { ath Score }\end{array}$ & $\begin{array}{c}\text { Quarter } 2 \\
\text { Math Grade }\end{array}$ & $\begin{array}{c}\text { Winter MAP } \\
\text { Reading } \\
\text { Score }\end{array}$ & $\begin{array}{c}\text { Quarter } 2 \\
\text { Reading } \\
\text { Grade }\end{array}$ & $\begin{array}{l}\text { Winter MAP } \\
\text { Languauge } \\
\text { Usage Score }\end{array}$ & $\begin{array}{l}\text { SRSS Total } \\
(0-21)\end{array}$ \\
\hline & 0 & 228 & 77 & 212 & 83 & 212 & 2 \\
\hline & 0 & 231 & 90 & 228 & 97 & 222 & 0 \\
\hline & 0 & 200 & 75 & 190 & 88 & 193 & 7 \\
\hline & 0 & 210 & 60 & 206 & 87 & 207 & 2 \\
\hline & 0 & 175 & 77 & 190 & 76 & 185 & 11 \\
\hline & 0 & 235 & 96 & 223 & 94 & 231 & 0 \\
\hline & 0 & 222 & 91 & 211 & 92 & 217 & 0 \\
\hline
\end{tabular}

